\title{
Binding of heterocycles by cyclohexanohemicucurbiturils and solid-phase extraction of sulfur-containing heterocycles from water
}

\author{
Tatsiana Shalima ${ }^{[a]}$, Kamini A. Mishra ${ }^{[a]}$, Sandra Kaabel ${ }^{[b]}$, Lukas Ustrnul[ ${ }^{[a]}$, Simona Bartkova ${ }^{[a]}$, Kaia \\ Tõnsuaadu ${ }^{[c]}$, Ivo Heinmaa ${ }^{[\mathrm{d}]}$ and Riina Aav*[a] \\ [a] Department of Chemistry and Biotechnology, Tallinn University of Technology, Akadeemia tee 15, 12618, Tallinn, Estonia, E-mail: riina.aav@taltech.ee \\ [b] Department of Chemistry, McGill University, 801 Sherbrooke St. West, Montréal, Québec H3A 0B8, \\ [c] Laboratory of Inorganic Materials, Institute of Materials and Environmental Technology, Tallinn University of Technology, Ehitajate tee 5, 12616, Tallinn, \\ Estonia
}

[d] Laboratory of Chemical Physics, National Institute of Chemical Physics and Biophysics, Akadeemia tee 23, 12618, Tallinn, Estonia

\section{Introduction}

Supramolecular chemistry, in particular, molecular recognition ${ }^{[1,2]}$, sensing ${ }^{[3-5]}$ and transport ${ }^{[6-9]}$ have promoted extensive research toward new applications. Out of many synthetic receptors, macrocycles with confined inner spaces distinctly demonstrate the hostguest interaction mechanism: a ring-shaped host possessing a cavity can form an inclusion complex with a suitably sized guest compound..$^{[10-15,2,16,17]}$ Hemicucurbiturils ${ }^{[18]}$ formed in templated single step oligomerization reactions, ${ }^{[19]}$ are single-bridged cucurbituriltype macrocycles that bear an electron-deficient hydrophobic cavity. The latter grants these macrocycles the ability to encapsulate anions, ${ }^{[18-20]}$ and also the formation of complexes with acids and some neutral species has been reported. In particular, unsubstituted hemicucurbit[ $n$ ] urils $(n=6,12)$ were efficiently used for liquid-liquid extraction and membrane transport of amino acid methyl esters, ${ }^{[21]}$ binding of hydroxyl-substituted Schiff bases ${ }^{[22]}$ and amino phenols, ${ }^{[23]}$ and ferrocene recognition. ${ }^{[24]}$ Cyclohexanohemicucurbit[n]urils (cycHC[n]) $(n=6,8,12)^{[25-29]}$ can bind anions ${ }^{[30,31]}$ and have been proven to form external complexes with Lewis and carboxylic acids in aprotic solvents. ${ }^{[26,32]}$ However, the binding of heterocycles by single-bridged cucurbiturils in solution has not been described previously. In the current study, we explore the binding of sulfur-, oxygen- and nitrogen-heterocycles with cycHCs (Figure 1).

Five- and six-membered heterocycles have relatively high electron density compared to carbocycles and may be able to occupy space within the eight-membered $\mathrm{cycHC}[8]$. S-heterocycles occur naturally ${ }^{[33]}$ and contribute to the distinct aroma of food, ${ }^{[34,35]}$ furthermore, tetrahydrothiophene is added as an odorant to natural gas.

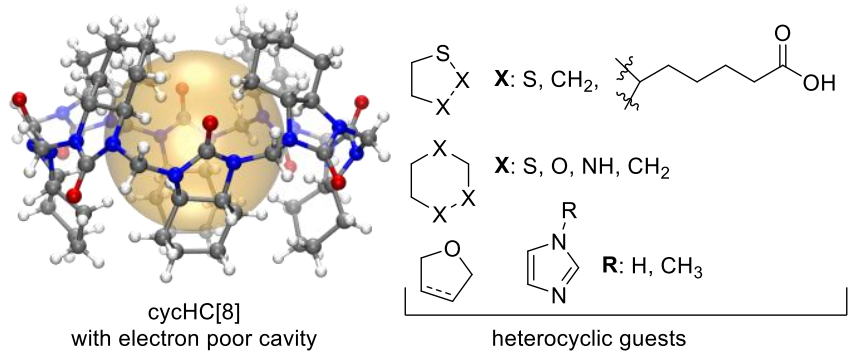

Figure 1. The structure of $\mathrm{cycHC}[8]$, highlighting the electron poor cavity where heterocyclic guests are bound. The scope of studied guests is shown on the right.

Many sulfur compounds bearing S-heterocycle fragments are bioactive ${ }^{[36]}$ and are exploited as insecticides and herbicides. ${ }^{[37]}$ For instance, $\alpha$-lipoic acid exhibits antioxidative properties and is used as a dietary supplement and a pharmaceutical agent. ${ }^{[38,39]}$ Unsubstituted S-containing heterocycles, such as 1,4-thioxane and 1,3-dithiolane, are found in meat ${ }^{[40]}$ and around chemical warfare dumping sites, some located in the Baltic Sea, where they are formed due to degradation of mustard gas. ${ }^{[41-43]}$

Commercially available solid-phase extraction (SPE) tubes ${ }^{[44]}$ and novel sorbent materials ${ }^{[45]}$ are used for the removal of S-heterocycles from water; however, such systems are designed to non-selectively retain all non-polar to moderately polar components. At the same time, to supplement the non-specific materials, selective sorbents can be employed. Surface-bound macrocyclic ligands illustrate one of the mechanisms of selective SPE based on molecular recognition, yet they are usually used for highly specific isolation of ionic species rather than neutral compounds. ${ }^{[46]}$ Thus far, cyclodextrins have been applied to form complexes with $\alpha$-lipoic acid, which is hydrophobic by nature, to enhance its solubility and bioavailability. ${ }^{[47-53]}$

Herein, we report that $\mathrm{cycHC}[8]$ encapsulates small neutral electron-rich organic molecules, namely, five- and six-membered heterocycles containing sulfur and oxygen atoms. This binding was characterized by ${ }^{1} \mathrm{H}$ NMR titration and isothermal titration calorimetry (ITC) in solution, and the structure of complexes was investigated by single crystal X-ray diffraction (SC-XRD), solid-state NMR (ssNMR), and thermogravimetric analysis (TGA). We compared the sorption properties of the homologs cycHC[6] and cycHC[8] 
and found that $\mathrm{cycHC}[8]$ is a promising sorbent material that can be applied for selective removal of S-containing hydrophobic neutral guests from water, such as 1,3-dithiolane and $\alpha$-lipoic acid.

\section{Results and Discussion}

\section{Inclusion complexes of S- and O-heterocycles with $\mathrm{cycHC}[8]$ in the solid state}

A series of suitably sized electron-rich $\mathrm{S}-, \mathrm{O}-$ and $\mathrm{N}$-heterocyclic compounds were crystallized via slow evaporation, and 2,5 dihydrofuran, tetrahydrofuran, 1,4-dioxane, 1,4-thioxane, and 1,3-dithiolane formed inclusion complexes with $(R, R)$-cycHC[8] upon cocrystallization (Figure 2 and SI pages S4-S13). The nitrogen-containing heterocycles, pyrrolidine and piperidine, and the largest explored guest, 1,4-dithiane, did not yield crystals of inclusion complexes with cycHC[8]. The packing of the 1,4-thioxane and 1,3dithiolane inclusion complexes with cycHC[8] gave rise to isomorphous ( $Z^{\prime}=4$ ) crystal structures (Figures S4, S5), in an arrangement previously unrecorded for $\mathrm{cycHC}[8]$ inclusion complexes. The packing of complexes involving oxygen-containing smaller heterocycles (Table 1) appears to be mainly directed by hydrogen bonding interactions with methanol so that the resulting crystal structures are isomorphous to each other (Figure S1-S3) and the previously published methanol solvate of $(R, R)$-cycHC[8]. ${ }^{[27]}$ However, clear features in the electron density map within the macrocycles in the respective host-guest complexes allowed to resolve the orientation of the encapsulated guests (Figure S6, S7). The smaller guest molecules, 2,5-dihydrofuran, tetrahydrofuran, and 1,4-dioxane, appear more disordered within $\mathrm{cycHC}[8]$, with a total site occupancy of the resolved disorder components limited to 50-75\%. The remaining electron density map had no clear features, making it impossible to resolve whether the diffuse component of the guest disorder also includes partial substitutional disorder from methanol. In contrast, the position of the larger sulfur-containing guests, 1,4-thioxane and 1,3dithiolane, is more conserved within the respective crystal structures (Figure S6, S7) with almost no diffuse component observed, indicating that these guests have fewer orientations available within the cycHC[8]. Notably, analyzing the disorder models of all inclusion complexes reveals a prevalent motif, where guests are oriented with heteroatoms close to the portals of cycHC[8] (Figure 2, see SI for full details). These are in several structures at a suitable distance (2.7-2.8 $\AA$ ) from a methanol molecule located at the portal of cychC[8] and can therefore potentially accept hydrogen bonds through the portals of the macrocycle.

Table 1. Respective sizes for the host and guests based on crystal structures and the packing coefficients (PC) of heterocycle@cycHC[8] complexes (A-E) (Figure 2, see SI for details).

\begin{tabular}{|c|c|c|c|c|}
\hline Host & $\begin{array}{l}V_{\text {cavity }} \\
\AA^{3 \text { [a] }}\end{array}$ & Guest & $V_{\text {guest }}, \AA^{3[b]}$ & Complex / PC ${ }^{[c]}$ \\
\hline \multirow{5}{*}{$\begin{array}{c}(R, R)- \\
\mathrm{cycHC}[8]\end{array}$} & \multirow{5}{*}{123} & 2,5-dihydrofuran & $62.2 \pm 0.2$ & A / 0.51 \\
\hline & & tetrahydrofuran & $66.0 \pm 0.5$ & B / 0.54 \\
\hline & & 1,4-dioxane & $74.6 \pm 0.7$ & C / 0.61 \\
\hline & & 1,4-thioxane & $84.7 \pm 0.6$ & D / 0.69 \\
\hline & & 1,3-dithiolane & $80.9 \pm 0.7$ & E / 0.66 \\
\hline
\end{tabular}

[a] The volume of $(R, R)-\mathrm{cycHC}[8]$ was determined in ref. ${ }^{[27]}[\mathrm{b}]$ The guest molecular volume was determined using the triangulated sphere model included in the Olex2 program package, ${ }^{[54]}$ in which all atoms are approximated as isotropic spheres defined by the default Cambridge Structural Database (CSD) van der Waals $(\mathrm{vdW})$ radii, and is given as an average of all guest molecules resolved in the asymmetric units of the respective crystal structures. [c] PC is the ratio between $V_{\text {guest }}$ to $V_{\text {cavity }}$ (host), reflecting the space filled by the encapsulated guest in the host cavity. ${ }^{[55]}$

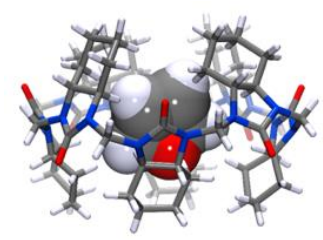

A

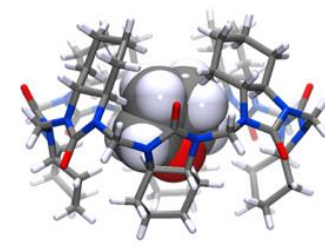

B

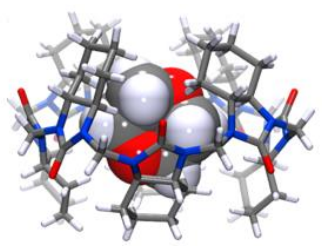

C

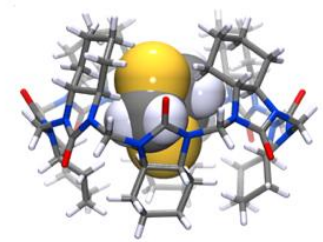

D

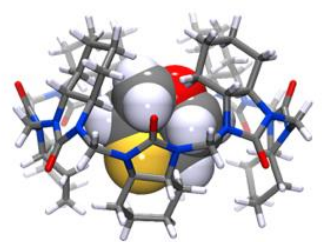

$\mathbf{E}$

Figure 2. Crystal structures of cycHC[8] inclusion complexes with neutral heterocycles: 2,5-dihydrofuran@cycHC[8] (A), tetrahydrofuran@cycHC[8] (B), 1,4-dioxane@cycHC[8] (C), 1,3-dithiolane@cycHC[8] (D), 1,4-thioxane@cycHC[8] (E). 
The latter would explain the observed conservation of this type of guest orientation motif. The guest molecules are tightly enwrapped within the cycHC[8], especially the largest sulfur-containing 1,3-dithiolane and 1,4-thioxane that fill close to $70 \%$ of the cavity volume (Table 1, complexes $\mathbf{D}$ and $\mathbf{E}$ ), indicating that guest binding and release must be accompanied with opening and closing the host portals. Similar conformational dynamics of $\mathrm{cycHC}[8]$ have been noted and computationally described previously in the binding of large anionic guests. ${ }^{[31]}$

\section{Screening of guest binding in solution}

Further, we evaluated host-guest complex formation in $\mathrm{CD}_{3} \mathrm{OD}$ solution. The scope of guests was expanded compared to the crystallographic studies and included two N-containing, four O-containing, and six S-containing heterocycles (Table 2 and Figure S8). Inclusion complex formation was followed by the chemical shift change of cycHC[8] proton $\mathrm{H}_{2 a x}$, positioned inside the cavity and becomes magnetically deshielded upon binding with the electron-rich guest. The change in cycHC[8] chemical shifts in the presence of 1,3-dithiolane is illustrated in Figure 3 and summarized in Table 2 for all the screened guests.

These studies revealed that S-containing five-membered heterocycles, 1,3-dithiolane and tetrahydrothiophene (Table 2, entries 1 and 2) caused a larger chemical shift change compared to the six-membered heterocycles; 1,4-thioxane, 1,4-dithiane, 1,3-dithiane and 1,4dioxane (Table 2, entries 3-6); which can be explained by a higher affinity and alarger population of complexed species formed with five-membered S-heterocycles. 1,3-dithiolane (Table 2, entry 1) induced the most prominent shift in the $\mathrm{H}_{2 a x}$ signal of cycHC[8] among all the compounds studied. A negligible shift was observed for tetrahydrofuran (Table 2, entry 7), while no shift was observed for 2,5dihydrofuran and $\mathrm{N}$-heterocycles, $\mathrm{N}$-methylimidazole, and imidazole (Table 2, entries 8, 10, and 11). Lipoic acid signals (Table 2, entry 9) overlapped with the characteristic cycHC[8] signal, and therefore its encapsulation could not be evaluated by ${ }^{1} \mathrm{H}$ NMR.
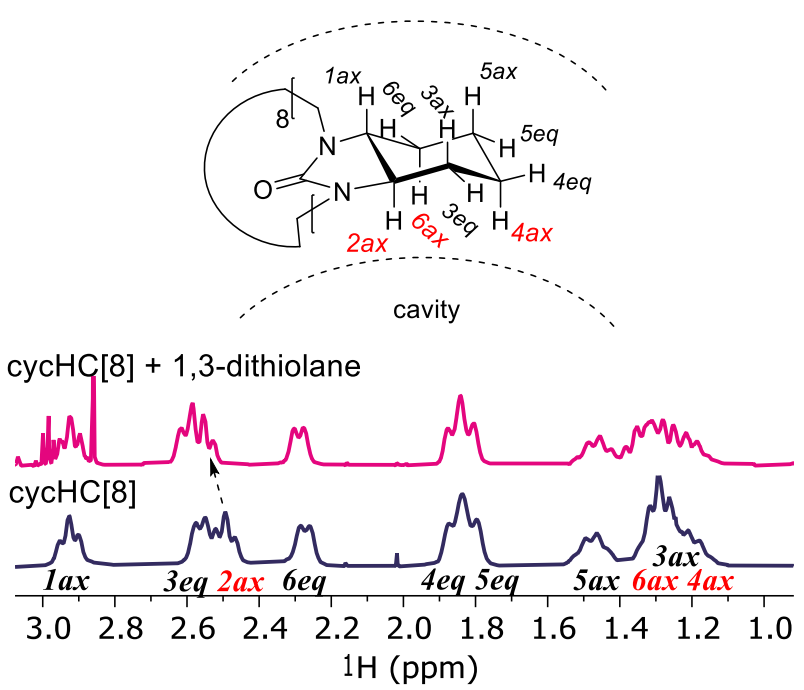

Figure 3. The structure of $\mathrm{cyHC}[8]$ with atom enumeration and ${ }^{1} \mathrm{H}$ NMR of cycHC[8] (lower spectrum) and cychC[8] with 60 eq. of $1,3-$ dithiolane (upper spectrum) in $\mathrm{CD}_{3} \mathrm{OD}$.

Table 2. Screening of guest binding in $\mathrm{CD}_{3} \mathrm{OD}$ using the ${ }^{1} \mathrm{H}$ NMR chemical shift change $(\Delta \delta)$ of $\mathrm{H}_{2 a x}$ signal of $3 \mathrm{mM}$ cychC[8] upon addition of 60 eq. of heterocycles.

\begin{tabular}{|c|c|c|c|c|c|}
\hline No & Guest & $\Delta \delta H_{2 a x}, p p m$ & No & Guest & $\Delta \delta H_{2 a x}, p p m$ \\
\hline 1 & & 0.064 & 7 & & 0.006 \\
\hline 2 & & 0.048 & 8 & & - \\
\hline 3 & & 0.030 & 9 & & $N A^{[a]}$ \\
\hline 4 & & 0.028 & 10 & & - \\
\hline 5 & & 0.013 & 11 & & - \\
\hline 6 & & 0.012 & & & \\
\hline
\end{tabular}

[a] Guest signals overlapped with cycHC[8] $\mathrm{H}_{2 a x}{ }^{1} \mathrm{H}$ NMR signal. 
The differences in the affinity of guests for binding to $\mathrm{cycHC}[8]$ are seemingly dependent on the hydrophobicity of the guests, which can be characterized by their partition coefficients (see Table S4). Nevertheless, all chemical shift changes were relatively small compared to those occurring upon the binding of anions, which caused up to a $0.6 \mathrm{ppm}$ chemical shift change in the $\mathrm{H}_{2 a x}$ signal. ${ }^{[31]}$ The binding of 1,3-dithiolane, which induced the largest chemical shift change in screening studies $(0.06 \mathrm{ppm})$, was further evaluated by NMR titration, and a weak association constant, $K=7.9 \pm 0.2 \mathrm{M}^{-1}$, was determined by a one-to-one binding model (Figures $4 \mathrm{a}, 4 \mathrm{~b}, \mathrm{SI}$ : Table S2, Figures S9-S10). ITC titration was performed to evaluate the thermodynamic parameters of binding 1,4-thioxane and 1,3dithiolane (Figures 4c, 4d, S11-S13, Table 3). The Gibbs free energy and association constant values for binding in $\mathrm{CH}_{3} \mathrm{OH}$ show that 1,3-dithiolane interacts with cycHC[8] more strongly than 1,4-thioxane, resulting in the association constants $13.1 \mathrm{M}^{-1}$ and $2.5 \mathrm{M}^{-1}$, respectively. Consequently, the binding of other $\mathrm{S}$ - and O-containing guests is expected to fall below the binding constant of 1,3dithiolane. The binding of both guests in methanol was enthalpically favorable and entropically unfavorable. A similar binding character was also observed upon the binding of chaotropic anions to $\mathrm{cycHC}[8]$ in protonic media. ${ }^{[31]}$ Even though chaotropicity is mainly attributed to the ionic species, there have been reports of chaotrope-like organic molecules studied in crystalline hydrates. ${ }^{[56]}$ As chaotropic character is exhibited in aqueous media, we further studied the binding of 1,3-dithiolane with cycHC[8] in a $1: 1$ mixture of $\mathrm{CH}_{3} \mathrm{OH}$ and $\mathrm{H}_{2} \mathrm{O}$. The results showed that the presence of water enhances the binding of the guest, raising the association constant to $65.6 \mathrm{M}^{-1}$. The enthalpy of binding was strongly increased in the presence of water, accompanied by a rise of the entropic penalty (Table 3). Herein we note that the quantitative study of the binding thermodynamics of these guests in pure water was not possible due to the very low water-solubility of cycHC[8].
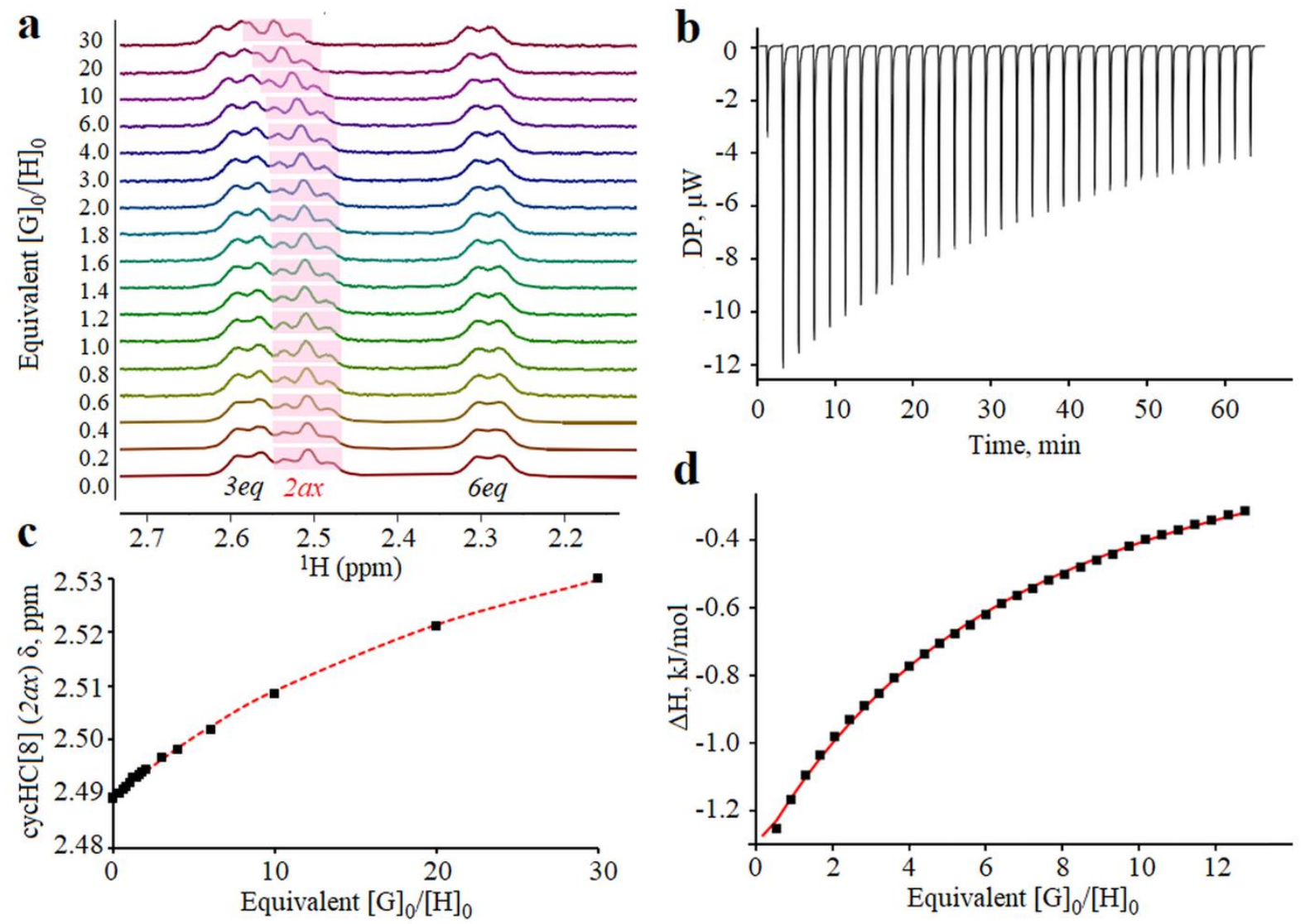

Figure 4. ${ }^{1} \mathrm{H}$ NMR and ITC titration for cycHC[8] binding with 1,3-dithiolane (a-spectra for NMR titration in $\mathrm{CD}_{3} \mathrm{OD}, \mathbf{b}-$ binding isotherm for $\mathrm{NMR}$ titration assuming the $1: 1$ binding model, $K=7.9 \pm 0.2 \mathrm{M}^{-1}, \mathbf{c}$ - raw thermogram for ITC measurement in $1: 1 \mathrm{CH}_{3} \mathrm{OH}: \mathrm{H}_{2} \mathrm{O}$ mixture, $\mathbf{d}-$ binding isotherm for ITC measurement using the "one set of sites" model).

Table 3. Thermodynamic parameters from ITC measurements for complexation with cycHC[8] for the $1: 1$ binding model. All energy values are given in $\mathrm{kJ} / \mathrm{mol}$.

\begin{tabular}{llllll}
\hline Guest & Solvent & $\boldsymbol{\Delta} \boldsymbol{H}^{\circ}$ & $-\boldsymbol{T} \boldsymbol{\Delta} \boldsymbol{S}^{\circ}$ & $\boldsymbol{\Delta} \boldsymbol{G}^{\circ}$ & $\boldsymbol{K}, \mathbf{M}^{-1}$ \\
\hline & $\mathrm{CH}_{3} \mathrm{OH}$ & $-13.7 \pm 1.2$ & 11.4 & -2.3 & $2.5 \pm 0.2$ \\
& $\mathrm{CH}_{3} \mathrm{OH}$ & $-9.8 \pm 0.6$ & 3.6 & -6.2 & $13.1 \pm 0.8$ \\
& $\begin{array}{c}\mathrm{CH}_{3} \mathrm{OH}: \mathrm{H}_{2} \mathrm{O} \\
(1: 1)\end{array}$ & $-20.4 \pm 0.9$ & 10.2 & -10.2 & $65.6 \pm 2.5$ \\
\hline
\end{tabular}




\section{Extraction of sulfur-containing heterocycles from water}

As outlined in the introduction, sulfur-containing heterocycles are found in pollutants, food aroma compounds, and bioactive substances; therefore, developing procedures for their selective removal from water is desirable. The low solubility of cycHC[8] in water and its ability to form inclusion complexes with heterocycles encouraged us to study the removal of sulfur-containing heterocycles from water via solid-phase extraction (SPE). To evaluate whether the ability of cycHC[8] to form inclusion complexes influences the sorption of heterocycles, we performed extraction experiments in parallel with the 6-membered homolog (cycHC[6]) of cycHC[8]. The cycHC[6] consists of the same monomers, so the hydrophobic properties of the outer surface are very similar to cycHC[8], but its cavity is much smaller $\left(35 \AA^{3}\right),{ }^{[27]}$ and thus it cannot accommodate the heterocycles studied. Hence, it served as a very informative analog for determining external or internal binding during extraction. $\mathrm{CycHC}[6]$ is also very poorly soluble in water and can be used in SPE as a solid sorbent. Textural properties of the sorbents, such as particle size and surface area, are important for the binding efficacy; therefore, prior to extraction experiments, the macrocyclic hosts were milled to obtain finer powders. Microscopic studies and image analysis of the samples (Figure 5) before and after milling showed that milling of both macrocycles produced particles of relatively uniform size, eliminating the larger aggregates and resulting in ca $80 \%$ of particles having a diameter under $5 \mu \mathrm{m}$ (Figures 6, S15-S17). In addition, the Brunauer-Emmett-Teller (BET) surface area of the solid extraction material was investigated by $\mathrm{N}_{2}$ adsorption-desorption analysis (SI, Table S3, and Figures S18-S23) and was found to be $6.03 \mathrm{~m}^{2} / \mathrm{g}$ and $9.02 \mathrm{~m}^{2} / \mathrm{g}$ for cycHC[6] and cycHC[8], respectively.

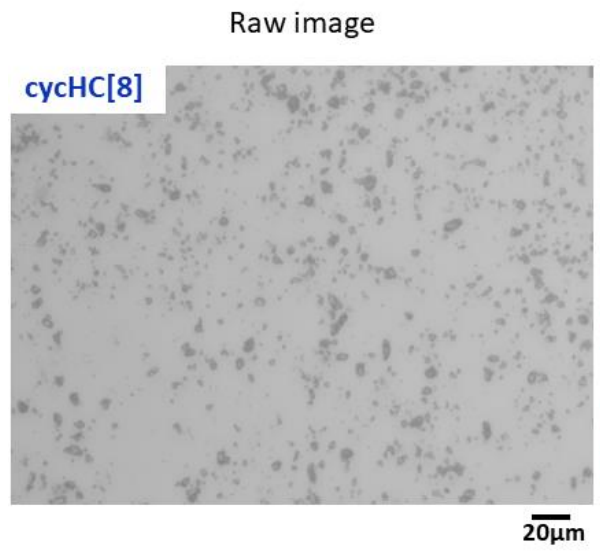

CellProfiler ${ }^{\top M}$ detected particles

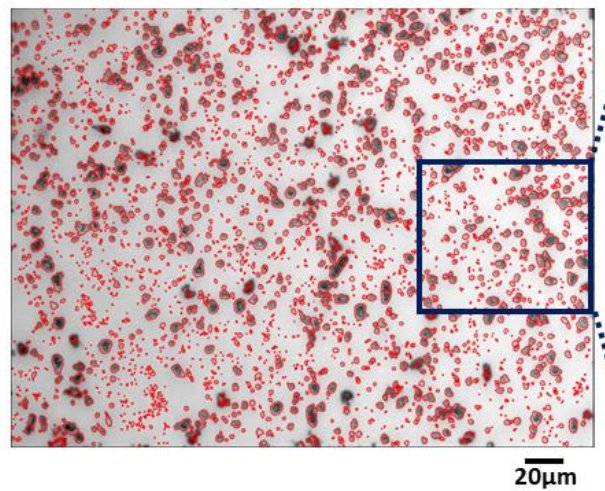

Zoom in on particles

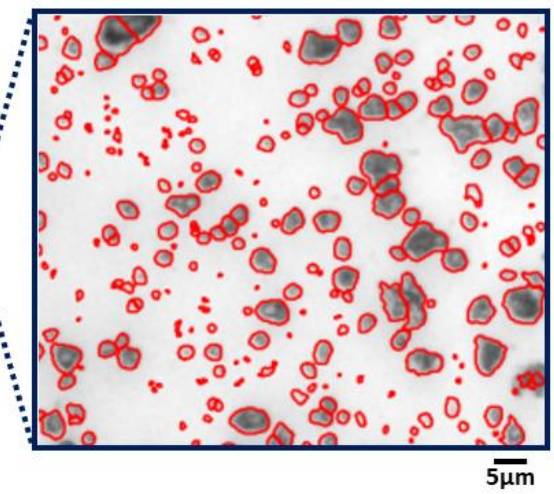

Figure 5. A representative image of $\mathrm{cycHC}$ [8] after milling. Left: Raw image. Middle: Image showing particles detected by CellProfiler. ${ }^{[57,58]}$ Right: Zoom for better visualization of detected particles. Red outlines indicate particles recognized and analyzed by the software.

According to this analysis of the sorbent material, and in the case where selectivity of binding would be caused by the availability of the surface area, cycHC homologs are expected to have similar extraction efficiencies.

SPE was performed for various heterocycles (imidazole, 1-methylimidazole, 1,4-dioxane, 1,4-thioxane, 1,3-dithiolane, $\alpha$-lipoic acid) by stirring solid $\mathrm{cycHC}[n]$ in an aqueous solution of each guest, and the change in the guest concentration was analyzed upon extraction. This change was assumed to be equal to the amount of host-guest complex formed. The results (Figure 7a, Table S5) demonstrate that roughly 5 molar excess of $\mathrm{cycHC}[n]$ gave negligible removal of O-containing 1,4-dioxane with cycHC[8] $(5 \%)$ and was inefficient in the case of $\mathrm{cycHC}[6]$, as well as for both macrocycles in the case of the $\mathrm{N}$-containing imidazole and 1-methylimidazole (Table S11). In contrast, $\mathrm{cycHC}[8]$ can efficiently extract the S-containing 1,3-dithiolane (78 \%) and moderately remove 1,4-thioxane (25\%), while cycHC[6] was considerably less efficient at removing these guests, $16 \%$ for 1,3 -dithiolane and only $3 \%$ of 1,4 -thioxane. These results indicate that the ability of $\mathrm{cycHC}[8]$ to form inclusion complexes with heterocycles enhances its extraction ability. Also, the extraction efficiency of cycHC[8] correlates with a stronger affinity toward S-containing heterocycles observed in our solution studies above.

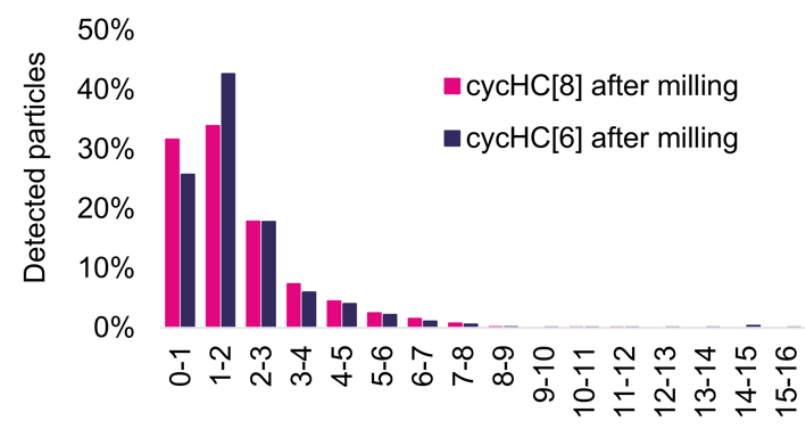

Diameter range, $\mu \mathrm{m}$

Figure 6. Comparison of size distribution of particles between milled samples of cychC[6] (dark blue) and cychC[8] (pink). 
In order to confirm that $\mathrm{cycHC}[8]$ can accommodate S-containing heterocycles in its cavity during SPE, thermogravimetric analysis of $\mathrm{cycHC}[n]$ sorbents enriched with 1,3-dithiolane during extraction was undertaken. Firstly, it was confirmed that the macrocycles exhibit thermal stability upon heating up to $200^{\circ} \mathrm{C}$, and no decomposition was observed. For the investigation of host-guest binding, solid macrocyclic $\mathrm{cycHC}[n]$, which retained guest molecules after the extraction procedure, were analyzed in parallel with pure 1,3-dithiolane (Figure S39). At a certain temperature, the latter would start to partially decompose, releasing volatile degradation products. The formation of characteristic mass-fragments of 1,3-dithiolane with $\mathrm{m} / \mathrm{z} 47$, corresponding to $\left[\mathrm{CH}_{2} \mathrm{SH}\right]^{-}$was followed during this analysis. The evolution profiles for pure 1,3-dithiolane and its host-guest complex with cycHC[6] are similar, indicating the decomposition products were released within the same temperature range and producing maximum ion current at $148^{\circ} \mathrm{C}$ and $138^{\circ} \mathrm{C}$, respectively.

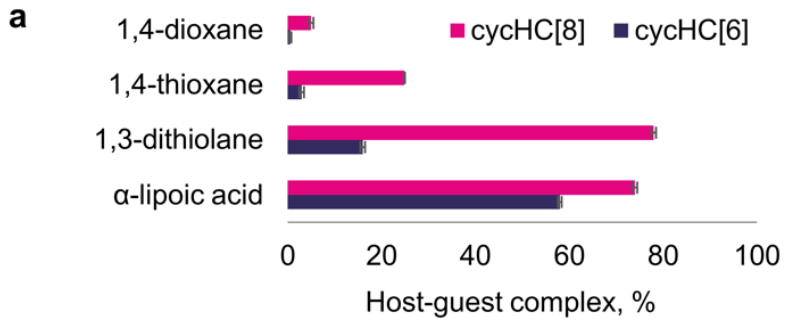

b

$$
\square(\mathrm{R}, \mathrm{R})-\mathrm{cycHC}[8] \quad \square(\mathrm{S}, \mathrm{S})-\mathrm{cycHC}[8]
$$

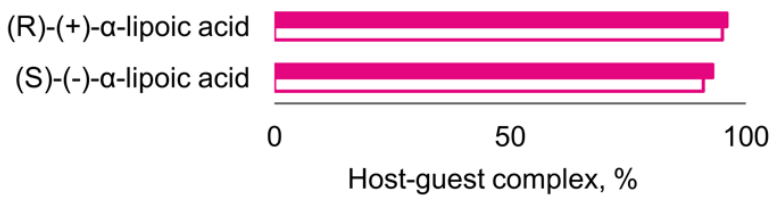

Figure 7. Efficiency of SPE of neutral guests from water conducted in presence of (a) 4-6 eq. of cycHC[6] and cycHC[8] (dark blue and pink, respectively) for 1,4dioxane, 1,4-thioxane, 1,3-dithiolane and $\alpha$-lipoic acid (error bars represent the standard deviation between parallel experiments $(n \geq 2)$ ); (b) 20 eq. of the $(R, R)$ - and $(S, S)$-cychC[8] (pink and white, respectively) for both enantiomers of $\alpha$-lipoic acid.

Meanwhile, the complex with cycHC[8] releases the characteristic degradation product at a significantly higher temperature, $189{ }^{\circ} \mathrm{C}$ (Figure S39A). This is regarded as evidence of 1,3-dithiolane encapsulation into the cycHC[8] cavity during the extraction procedure, which leads to a higher affinity toward this guest.

The results of the extraction of the larger and more hydrophobic a-lipoic acid are much more similar between cycHC[6] and cycHC[8] (Figure $7 \mathrm{a}$ ). With 5 eq. cycHC[8], $74 \%$ of $\alpha$-lipoic acid was removed from the aqueous solution. A higher excess (20 eq.) of the macrocyclic host, cycHC[8], resulted in the removal of over $90 \%$ of the a-lipoic acid from the water, but unfortunately, this occurred with no enantioselectivity (Figure 7b, Tables S6-S7). In a comparative study with 5 eq. excess of cycHC[6], $58 \%$ of $\alpha$-lipoic acid was extracted, which differs only by ca $16 \%$ for the relative efficiency gained by cycHC[8] under similar conditions. This relatively small selectivity difference indicates the very different character of a-lipoic acid binding to cycHCs, compared to 1,3-dithiolane. To better understand the interaction of $\alpha$-lipoic acid with cycHC[8] and cycHC[6], complex formation was studied by solid-state NMR (ssNMR) spectroscopy. Recently, complex formation with cucurbit[7]uril via mechanochemical agitation followed by ssNMR has also been demonstrated by Masson and Kaleta. ${ }^{[59]}$ The macrocycle and $\alpha$-lipoic acid in a 1:1 molar ratio were milled together in the presence of a small amount of water $(\eta=0.3 \mu \mathrm{l} / \mathrm{mg})$, that is, modeling the binding during the extraction process at the solute-solid interphase. The milled sample obtained and a mixture of $\alpha$-lipoic acid and $\mathrm{cycHC}[n]$ stirred together without applying additional mechanical force were investigated by ${ }^{13} \mathrm{C}$ ssNMR spectroscopy (Figures S40-S46). Figure 8 illustrates the changes induced upon binding $\alpha$-lipoic acid with $\mathrm{cycHC}$ [8]. Analogous data were obtained for the complexation with cycHC[6] (Figures S45-S46). Overall, a-lipoic acid has clearly visible sharp signals in the range of 32-42 ppm in the sample, which was not milled, while in the milled sample, these signals were broadened, suggesting a change in the close environment of the lipoic acid either due to interaction with the water or the cycHC[8]. Additionally, the carboxylic group signal of C10' shifts from $183 \mathrm{ppm}$ to $175 \mathrm{ppm}$ (C10"), indicating additional shielding of the carboxyl groups, which may be caused by disaggregation of the $\alpha$-lipoic acid and complexation with cycHC[8]. All eight monomers of cycHC[8] are symmetry-related, and though they have the same chemical shifts, for this reason, changes in chemical shifts of cycHC[8] are hard to observe upon the binding of 1 equivalent of a-lipoic acid. Nevertheless, there are noticeable changes in the cycHC[8] signal intensities, as shown in the regions highlighted by dashed pink rectangles in Figure 8 . The intensities of several of the carbons $(\mathrm{C} 1$, C8, C3, and C5) have been enhanced relative to others, which could point to decreasing conformational flexibility. Very similar changes in the spectra of $\alpha$-lipoic acid and cycHC[6] (Figures S47-S48) were observed in samples prepared similarly to those described above. Therefore, based on this data, we propose that the interaction between the $\alpha$-lipoic acid and cycHC[ $n]$ s occurs from the outside surface of the macrocycles. The small difference in the extraction efficiency is caused by their small particle size and surface area differences. A clear tendency is observed throughout the extraction experiments: the hydrophobic unsubstituted S-containing heterocycles can be efficiently removed from water via inclusion complex formation with cycHC[8]. Substituents on highly hydrophobic $\alpha$-lipoic acid hinder the formation of inclusion complexes, and therefore both cycHCs act similarly via external binding during extraction. On the contrary, the extraction of less hydrophobic $\mathrm{O}$ - and $\mathrm{N}$-heterocycles by cycHCs is low or negligible. 


\section{Regeneration of the sorbent}

The reusability of the sorbent was studied, and it was found that the solid macrocyclic material, cycHC[8], can be used multiple times. A simple washing and drying procedure, followed by milling, allows the reactivation of the surface of the sorbent for future use without significant loss in binding efficiency for at least four cycles (Figure 9, Table S12). It was also confirmed that the cycHC[8] sorbent content remained unchanged (Figure S47).

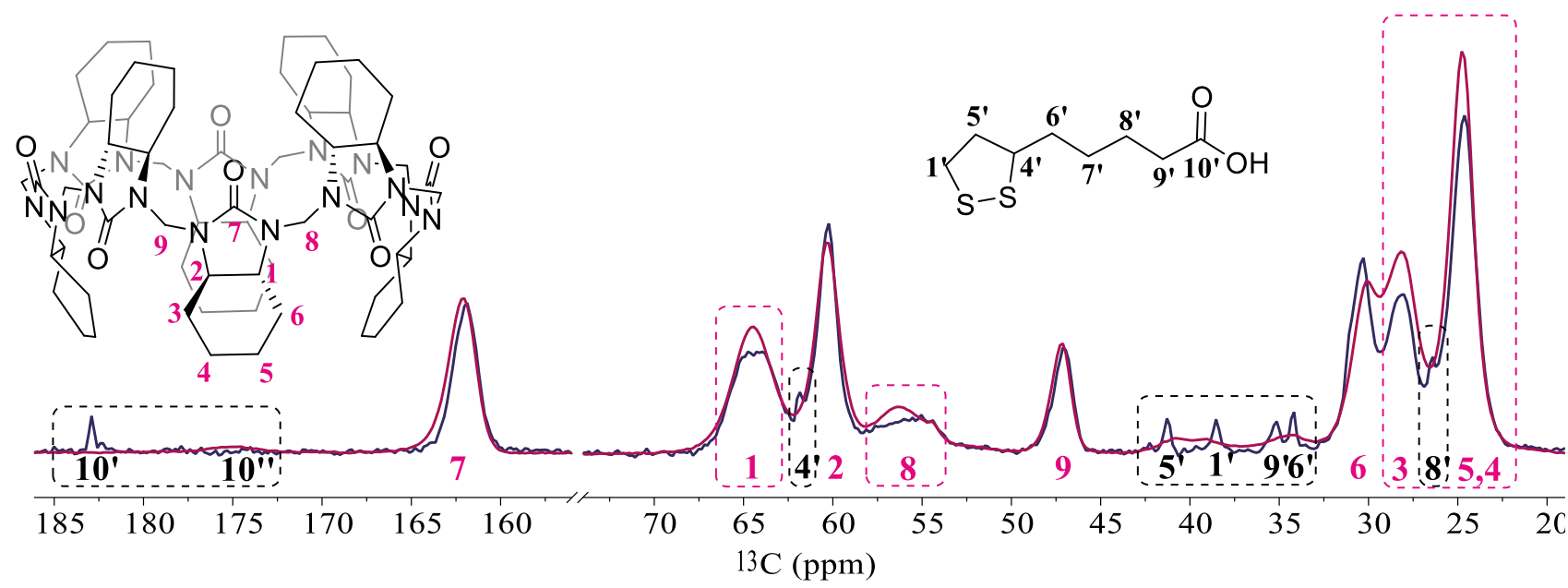

Figure $8 .{ }^{13} \mathrm{C}$ NMR spectra of $1: 1$ mixture of $\mathrm{cycHC}[8]$ and $\alpha$-lipoic acid after milling (red) versus their mixture without milling (dark blue).

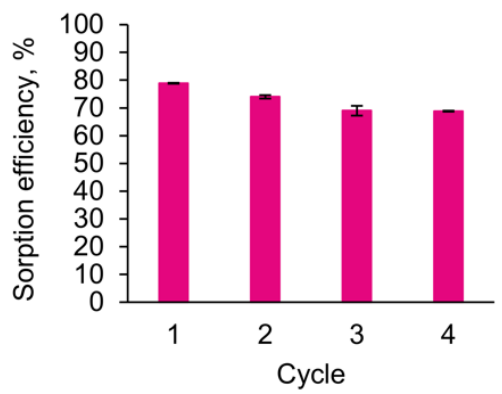

Figure 9. Sorption performance of $\mathrm{cycHC}[8]$ during four 1,3-dithiolane extraction cycles. Error bars represent the standard deviation between parallel experiments $(n=3)$.

\section{Conclusion}

There is strong evidence for the formation of inclusion complexes between cycHC[8] and S- and O-containing heterocyclic guests in the solid state and solution, which was thoroughly investigated by SC-XRD and ${ }^{1} \mathrm{H}$ NMR. Encapsulation of 5- and 6-membered electronrich heterocyclic guests is mostly related to their size, which must be complementary with the host cavity, and the hydrophobic effect is one of the driving forces of the interaction. The latter explains why complexation with polar $\mathrm{N}$-containing heterocycles was not observed. For 1,3-dithiolane, which induced the most prominent change in the characteristic ${ }^{1} \mathrm{H}$ NMR signal of cycHC[8], association constants were determined in methanol and a methanol-water mixture. From these results, it was evident that complex formation is even more favorable in water and the thermodynamic characteristics resembled the binding of chaotropic anions.

Since the $\operatorname{cycHC}[n]$ are almost insoluble in water, they can serve as solid sorbent material for SPE of heterocycles from aqueous solutions. $\mathrm{CycHC}[8]$ has expressed the highest affinity toward the most hydrophobic S-containing heterocycles and was successfully applied for their selective extraction via inclusion complex formation, which was proven by TGA experiments. However, in the case of $\alpha$-lipoic acid, the evidence collected by ${ }^{13} \mathrm{C}$ solid-state NMR shows that hydrophobic $\alpha$-lipoic acid forms an external complex with both $\mathrm{cycHC}[6]$ and $\mathrm{cycHC}[8]$, and its extraction results suggest that the binding efficiency correlated with the small differences in particle size and surface area.

Solid cycHC[8] can be used for selective removal of neutral hydrophobic S-containing heterocycles, which can be found in environmental samples, from water. In addition, $\mathrm{cycHC}[n]$ proved to efficiently bind hydrophobic substituted heterocycles in the example of biologically active $\alpha$-lipoic acid. Crucially, the solid macrocyclic sorbent material can be reused, which makes it a candidate for applications in selective SPE systems or the removal of target compounds from water, based on molecular recognition properties. 


\section{Experimental Section}

Detailed descriptions of the experiments are provided in the SI.

Single crystals of the host-guest complexes were obtained from saturated $(\mathrm{R}, \mathrm{R})$-cycHC[8] solutions in methanol by adding $20 \mu \mathrm{l}$ of the respective guest compound. Single crystal X-ray diffraction data were collected at $123 \mathrm{~K}$ on a Rigaku Compact HomeLab diffractometer, equipped with a Saturn $944 \mathrm{HG}$ CCD detector and an Oxford Cryostream cooling system, using monochromatic Cu-Ka radiation (1.54178 $\AA$ ) from a MicroMax ${ }^{\mathrm{TM}}-003$ sealed tube microfocused X-ray source. The crystallographic data are deposited with the Cambridge Crystallographic Data Centre (CCDC 2069875-2069879) and can be obtained free of charge via www.ccdc.cam.ac.uk/data request/cif.

Complexation-induced shifts of $(R, R)$-cycHC[8] $(3 \mathrm{mM})$ were investigated by ${ }^{1} \mathrm{H}$ NMR spectroscopy in $\mathrm{CD}_{3} \mathrm{OD}$ solution upon addition of 60 eq. of each guest compound. The association constant for complexation with 1,3-dithiolane was determined by NMR titration. ${ }^{1} \mathrm{H} \mathrm{NMR}(400 \mathrm{MHz})$ spectra in solution were recorded on a Bruker Avance III spectrometer.

ITC was performed on MicroCal PEAQ-ITC calorimeter (Malvern GE Healthcare Life Sciences) using a $200 \mu \mathrm{L}$ calorimetric cell and a $40 \mu \mathrm{L}$ syringe MicroCal PEAQ-ITC analysis software (Malvern) was used for data analysis.

Extraction of heterocycles from aqueous solutions

Prior to analysis and extraction experiments, $\mathrm{cycHC}[\mathrm{n}]$ was milled in an FTS-1000 shaker mill at a frequency of $30 \mathrm{~Hz}$ by using a $14 \mathrm{~mL} \mathrm{ZrO}-\mathrm{coated}$ grinding jar charged with $3 \times 7 \mathrm{~mm} \mathrm{ZrO}_{2}$ milling balls for $30 \mathrm{~min}$.

Neutral guest compounds were extracted from aqueous solutions by the milled solid cycHC[n]. A Mettler Toledo AB204-2 analytical balance (precision $0.1 \mathrm{mg}$ ) and a Radwag MYA 11.4Y microbalances (precision $0.006 \mathrm{mg}$ ) were used in sample preparation. The test samples included blank suspensions with the macrocyclic hosts (cycHC[n]), control solutions of the guests, and experimental suspensions of hosts in the presence of the guests. The cycHC[n] was taken in roughly 5 or 20 molar excess to the amount of guest. Within one experiment, all test suspensions were treated identically: mixed on either a Vortex-Genie 2 mixer or a Stuart magnetic stirrer for 30-60 min, followed by separation of heterogeneous mixtures using a Hettich Universal 32R centrifuge or Regenerated cellulose (RC) membrane syringe filters, and analysis of the liquid phase (experimental solution). The concentrations of the guest before and after the extraction process (control and experimental solutions, respectively) were determined by either HPLC-UV or UV spectrophotometry (SI pages S24-S34). HPLC determination of guest compounds was carried out on an Agilent 1200 Series HPLC system equipped with a multiple wavelength detector (MWD), a Macherey-Nagel Nucleoshell RP18 column $(150 \times 3.0 \mathrm{~mm}, 2.7 \mu \mathrm{m})$ or a Phenomenex Kinetex XB-C18 column $(150 \times 4.6 \mathrm{~mm}, 2.6 \mu \mathrm{m})$. UV absorption of guest compound solutions was measured by a Jasco V-730 dual-beam spectrophotometer and a Varian Cary 50 UV-Vis spectrophotometer.

Surface area analysis of the milled cycHC[n] was performed on a KELVIN $1040 / 1042$ Sorptometer at $150{ }^{\circ} \mathrm{C}$ with $\mathrm{N}_{2}$ as an adsorptive gas and He as the carrier gas. The obtained data were processed using Kelvin 1042 V3.12 software. A microscopic investigation of cycHC[n] particle sizes and their distribution was performed before and after milling. The solid samples were examined with an Olympus BX61 microscope. The acquired images were further analyzed by CellProfiler (version 4.0.3) software. ${ }^{[57,58]}$

The complexation between cycHC[n] and 1,3-dithiolane after extraction was studied by simultaneous thermogravimetry and differential thermal analysis coupled with evolved gas mass spectrometric analysis (TG-DTA/EGA-MS). The measurements were performed in an apparatus consisting of a SetSysEvo 1600 thermal analyzer and an OmniStar quadrupole mass spectrometer (gastight high-sensitivity ion source at an ion source voltage of $150 \mathrm{~V}$ ). The binding of $\alpha$-lipoic acid by $\mathrm{cycHC}[\mathrm{n}]$ was studied by ${ }^{13} \mathrm{C}$ solid-state CPMAS NMR spectroscopy. The spectra were acquired on a Bruker AVANCE-II spectrometer under a $14.1 \mathrm{~T}$ magnetic field $\left({ }^{13} \mathrm{C}\right.$ resonance frequency $\left.150.91 \mathrm{MHz}\right)$ using a home-built MAS probe for $25 \times 4 \mathrm{~mm} \mathrm{Si} \mathrm{N}_{4}$ rotors.

The regeneration of sorbent $(R, R)$-cycHC[8] was investigated by comparing its extraction efficiency after four sorption-desorption cycles. The sorption step was performed analogously to the extraction procedure. The desorption step included rinsing the material with distilled $\mathrm{H}_{2} \mathrm{O}$, drying for 6 hours at $114{ }^{\circ} \mathrm{C}$ in the oven, followed by drying for 3 hours under vacuum ( $25 \mathrm{psi}$ or $1.72 \mathrm{bar}$ ). The dried macrocycle was milled according to the general procedure and used for the subsequent cycle.

\section{Acknowledgments}

This work was financially supported by Estonian Research Council Grants No. PRG399, MOBJD556 and MOBJD592, the ERDF (CoE 2014-2020.4.01.15-0013, and CoE TK134), H2020-FETOPEN (828779 (INITIO)), and European Regional Research Council, Grant No. TK134. The authors would also like to thank Elina Suut and Jagadeesh Varma Nallaparaju for the synthesis of $c y c H C[n]$, Dr. Heidi Lees, and Piia Jõul for providing the S-containing heterocyclic compounds used for crystallization and extraction experiments, and Mai Uibu for BET analysis.

\section{References}

[1] C.-L. Deng, S. L. Murkli, L. D. Isaacs, Chem. Soc. Rev. 2020, 49, 7516-7532.

[2] S. J. Barrow, S. Kasera, M. J. Rowland, J. del Barrio, O. A. Scherman, Chem. Rev. 2015, 115, 12320-12406.

[3] R. Pinalli, A. Pedrini, E. Dalcanale, Chem. Soc. Rev. 2018, 47, 7006-7026.

[4] R. Kubota, Y. Sasaki, T. Minamiki, T. Minami, ACS Sens. 2019, 4, 2571-2587.

[5] T. L. Mako, J. M. Racicot, M. Levine, Chem. Rev. 2019, 119, 322-477.

[6] J. Mayr, C. Saldías, D. D. Díaz, Chem. Soc. Rev. 2018, 47, 1484-1515.

[7] A. S. Braegelman, M. J. Webber, Theranostics 2019, 9, 3017-3040.

[8] J. T. Davis, P. A. Gale, R. Quesada, Chem. Soc. Rev. 2020, 49, 6056-6086.

[9] J. Liu, X. Ding, Y. Fu, C. Xiang, Y. Yuan, Y. Zhang, P. Yu, Eur. J. Med. Chem. 2021, 212, 113105

[10] Z. Liu, S. K. M. Nalluri, J. F. Stoddart, Chem. Soc. Rev. 2017, 46, 2459-2478.

[11] J. Phatsawee, O. Noriko, L. Thorsteinn, Int. J. Pharm 2018, 535, 274-284.

[12] M. Majd, M. Yazdanpanah, M. R. Bayatloo, S. Nojavan, Talanta 2021, 229, 122296.

[13] Md. T. Sikder, Md. M. Rahman, Md. Jakariya, T. Hosokawa, M. Kurasaki, T. Saito, Chem. Eng. J. 2019, 355, 920-941.

[14] G. Crini, Chem. Rev. 2014, 114, 10940-10975.

[15] R. Kumar, A. Sharma, H. Singh, P. Suating, H. S. Kim, K. Sunwoo, I. Shim, B. C. Gibb, J. S. Kim, Chem. Rev. 2019, 119, 9657-9721.

[16] E. Masson, X. Ling, R. Joseph, L. Kyeremeh-Mensah, X. Lu, RSC Adv. 2012, 2, 1213-1247.

[17] K. I. Assaf, W. M. Nau, Chem. Soc. Rev. 2014, 44, 394-418. 
[18] N. N. Andersen, M. Lisbjerg, K. Eriksen, M. Pittelkow, Isr. J. Chem. 2018, 58, 435-448.

[19] S. Kaabel, R. Aav, Isr. J. Chem. 2018, 58, 296-313.

[20] T. Lizal, V. Sindelar, Isr. J. Chem. 2018, 58, 326-333.

[21] E. I. Cucolea, H.-J. Buschmann, L. Mutihac, Supramol. Chem. 2016, 28, 727-732.

[22] X.-Y. Jin, F. Wang, H. Cong, Z. Tao, J. Incl. Phenom. Macrocycl. Chem. 2016, 86, 249-254.

[23] X.-Y. Jin, F. Wang, H. Cong, Z. Tao, J. Incl. Phenom. Macrocycl. Chem. 2016, 86, 241-248.

[24] B. Wittmann, F. A. Wenzel, S. Wiesneth, A. T. Haedler, M. Drechsler, K. Kreger, J. Köhler, E. W. Meijer, H.-W. Schmidt, R. Hildner, J. Am. Chem. Soc. 2020, 142, 8323-8330.

[25] Y. Li, L. Li, Y. Zhu, X. Meng, A. Wu, Cryst. Growth Des. 2009, 9, 4255-4257.

[26] R. Aav, E. Shmatova, I. Reile, M. Borissova, F. Topić, K. Rissanen, Org. Lett. 2013, 15, 3786-3789.

[27] E. Prigorchenko, M. Öeren, S. Kaabel, M. Fomitšenko, I. Reile, I. Järving, T. Tamm, F. Topić, K. Rissanen, R. Aav, Chem. Commun. 2015, 51, 10921-10924.

[28] E. Prigorchenko, S. Kaabel, T. Narva, A. Baškir, M. Fomitšenko, J. Adamson, I. Järving, K. Rissanen, T. Tamm, R. Aav, Chem. Commun. 2019, 55, 93079310.

[29] K. A. Mishra, J. Adamson, M. Öeren, S. Kaabel, M. Fomitšenko, R. Aav, Chem. Commun. 2020, 56, 14645-14648.

[30] M. Öeren, E. Shmatova, T. Tamm, R. Aav, Phys. Chem. Chem. Phys. 2014, 16, 19198-19205.

[31] S. Kaabel, J. Adamson, F. Topić, A. Kiesilä, E. Kalenius, M. Öeren, M. Reimund, E. Prigorchenko, A. Lõokene, H. J. Reich, K. Rissanen, R. Aav, Chem. Sci. 2017, 8, 2184-2190.

[32] L. Ustrnul, S. Kaabel, T. Burankova, J. Martõnova, J. Adamson, N. Konrad, P. Burk, V. Borovkov, R. Aav, Chem. Commun. 2019, 55, 14434-14437.

[33] K. Mahadevan, L. Farmer, J. Agric. Food Chem. 2006, 54, 7242-7250.

[34] D. S. Mottram, H. R. Mottram, in Heteroatomic Aroma Compounds, ACS, 2002, pp. 73-92.

[35] S. Schoenauer, P. Schieberle, J. Agric. Food Chem. 2018, 66, 4189-4199.

[36] T. Rezanka, M. Sobotka, J. Spizek, K. Sigler, Antiinfect. Agents Med. Chem 2006, 5, 187-224.

[37] C. Lamberth, H. Walter, F. M. Kessabi, L. Quaranta, R. Beaudegnies, S. Trah, A. Jeanguenat, F. Cederbaum, Phosphorus. Sulfur. Silicon Relat. Elem. 2015, $190,1225-1235$

[38] L. Rochette, S. Ghibu, C. Richard, M. Zeller, Y. Cottin, C. Vergely, Mol. Nutr. Food Res. 2013, 57, 114-125.

[39] B. Salehi, Y. Berkay Yılmaz, G. Antika, T. Boyunegmez Tumer, M. Fawzi Mahomoodally, D. Lobine, M. Akram, M. Riaz, E. Capanoglu, F. Sharopov, N. Martins, W. C. Cho, J. Sharifi-Rad, Biomolecules 2019, 9, 356.

[40] V. Garbusov, G. Rehfeld, G. Wölm, R. V. Golovnja, M. Rothe, Mol. Nutr. Food. Res. 1976, 20, $235-241$.

[41] B. T. Røen, E. Unneberg, J. Aa. Tørnes, E. Lundanes, J. Chromatogr. A 2010, 1217, 2171-2178.

[42] R. Magnusson, T. Nordlander, A. Östin, J. Chromatogr. A 2016, 1429, 40-52.

[43] M. Söderström, CHEMSEA WP3. Summary of Chemical Analysis of Sediment Samples, Http://Chemsea.Eu/ (Accsessed 16.04.2021), 2014.

[44] H. Lees, M. Vaher, M. Kaljurand, Electrophoresis 2017, 38, 1075-1082.

[45] P. Jõul, M. Vaher, M. Kuhtinskaja, Chemosphere 2018, 198, 460-468.

[46] I. M. M. Rahman, Z. A. Begum, H. Hasegawa, Microchem. J. 2013, 110, 485-493.

[47] T. Lin-Hui, P. Zheng-Zhi, Y. Ying, J. Incl. Phenom. Macrocycl. Chem. 1995, 23, 119-126.

[48] H. Maeda, T. Onodera, H. Nakayama, J. Incl. Phenom. Macrocycl. Chem. 2010, 68, 201-206.

[49] H. Takahashi, Y. Bungo, K. Mikuni, Biosci. Biotechnol. Biochem. 2011, 75, 633-637.

[50] C. P. Racz, S. Santa, M. Tomoaia-Cotisel, G. Borodi, I. Kacso, A. Pirnau, I. Bratu, J. Incl. Phenom. Macrocycl. Chem. 2013, 76, 193-199.

[51] M. R. Caira, S. A. Bourne, B. Mzondo, Molecules 2017, 22, 866

[52] N. Ikuta, H. Sugiyama, H. Shimosegawa, R. Nakane, Y. Ishida, Y. Uekaji, D. Nakata, K. Pallauf, G. Rimbach, K. Terao, S. Matsugo, Int. J. Mol. Sci. 2013 14, 3639-3655.

[53] A. Celebioglu, T. Uyar, J. Agric. Food Chem. 2019, 67, 13093-13107.

[54] O. V. Dolomanov, L. J. Bourhis, R. J. Gildea, J. A. K. Howard, H. Puschmann, J. Appl. Crystallogr. 2009, 42, 339-341.

[55] S. Mecozzi, Jr. Rebek Julius, Chem. Eur. J. 1998, 4, 1016-1022.

[56] Ł. Dobrzycki, P. Socha, A. Ciesielski, R. Boese, M. K. Cyrański, Cryst. Growth Des. 2019, 19, 1005-1020.

[57] A. E. Carpenter, T. R. Jones, M. R. Lamprecht, C. Clarke, I. H. Kang, O. Friman, D. A. Guertin, J. H. Chang, R. A. Lindquist, J. Moffat, P. Golland, D. M. Sabatini, Genome Biol. 2006, 7, R100.

[58] C. McQuin, A. Goodman, V. Chernyshev, L. Kamentsky, B. A. Cimini, K. W. Karhohs, M. Doan, L. Ding, S. M. Rafelski, D. Thirstrup, W. Wiegraebe, S. Singh, T. Becker, J. C. Caicedo, A. E. Carpenter, PLoS Biol. 2018, 16, e2005970.

[59] M. Dračínský, C. S. Hurtado, E. Masson, J. Kaleta, Chem. Commun. 2021, 57, 2132-2135. 